\title{
Características clínicas y epidemiológicas de la infección por VIH en inmigrantes latinoamericanos versus chilenos: estudio comparativo en un centro de atención de Santiago a partir de registros de 2003-2013
}

\author{
M. Fernanda Rodríguez, Marcelo Wolff y Claudia Cortés
}

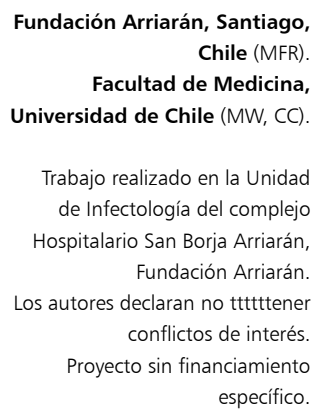

Fundación Arriarán, Santiago Chile (MFR)

Facultad de Medicina Universidad de Chile (MW, CC).

Trabajo realizado en la Unidad de Infectología del complejo Hospitalario San Borja Arriarán Fundación Arriarán. Los autores declaran no ttttttene conflictos de interés.

Proyecto sin financiamiento específico.

Recibido: 2 de junio de 2014 Aceptado: 27 de agosto de 2014

Correspondencia a: María Fernanda Rodríguez M. fdarodriguez@yahoo.com

Clinical and epidemiological characteristics of HIV infection in Latin-American immigrants. Comparative analysis from clinical records in a clinical care centre in Santiago in the last decade

Background: There has been an increasing number of immigrants to Chile in the last years, especially from South American countries. The phenomenon of immigration and its consequences has been studied by international literature, and different health care needs have been reported for this group as compared with local population. In Chile this phenomenon is poorly studied and HIV prevention campaigns are focused on national population needs. Objective: To determine baseline clinical and epidemiological characteristics of the HIV infection in Latin-American immigrants presenting to a referral HIV clinical care centre between the years 2003-2013. Methods: Retrospective analysis. Baseline characteristics of Latin-American immigrants at admission to the infectious disease unit were compared to a peered group of Chileans in the same unit. Results: There was an increase in the number of immigrants trough out the observation period. Foreigners presented larger proportion of women ( $26 \%$ vs. $9 \%$ ) and heterosexual conduct as compared to nationals ( $37 \%$ vs $22 \%)$. The majority of immigrants came from Peru (55\%) and Colombia (12\%). Conclusions: There were significant differences in regards to gender and sexual behavior. This brings up the need to address different prevention strategies with more emphasis in women and heterosexual population in this vulnerable group.

Key words: HIV, emigrants and immigrants, Latin America, vulnerable populations.

Palabras clave: VIH, emigrantes e inmigrantes, América Latina, poblaciones vulnerables.

\section{Introducción}

$\mathrm{E}$ 1 cambio en las características migratorias de las poblaciones genera no sólo un cambio sociocultural, sino también un cambio en la realidad epidemiológica de los países ${ }^{1}$.

Chile ha sido y se mantiene como un país generador de emigrantes. No obstante, en los últimos años ha existido un aumento de las inmigraciones, alcanzando el año 2007 la mayor cifra desde 1950. En las últimas décadas del siglo XX prevalece la migración proveniente de países fronterizos. Esta tendencia se consolida con el advenimiento de la democracia y estabilidad en Chile ${ }^{2}$.

Según el censo poblacional de 2002, último censo disponible, la cantidad de extranjeros residentes en Chile es de 184.464 personas. (1,2\% de la población general). El $67,8 \%$ de ellos son de origen sudamericano ${ }^{3}$. En el Área Metropolitana la superioridad numérica de los peruanos es manifiesta y estos presentan además la mayor tasa de crecimiento ${ }^{4,5}$.
Tres tendencias caracterizan a los inmigrantes en Chile: predominio femenino creciente, mayor nivel de educación y concentración en edades de mayor actividad laboral que las generaciones precedentes ${ }^{6}$.

La literatura sobre las condiciones de vida y acceso a la salud de la población inmigrante en Chile es escasa. La Encuesta de Caracterización Socioeconómica Nacional (CASEN) 2006 sugiere que los inmigrantes presentan una alta polaridad en cuanto al status socioeconómico ${ }^{7}$. Según datos de un estudio de casos del Ministerio del Interior, se encuentran en situación administrativa irregular $16 \%$ de ellos $^{8}$. Según un estudio derivado de datos provenientes de la encuesta CASEN 2006 este porcentaje podría alcanzar a $35 \%{ }^{9}$. El Ministerio del Interior reconoce que existe un desarrollo pendiente en temas laborales, de vivienda, victimización y discriminación, que afecta especialmente a mujeres e inmigrantes en situación irregular ${ }^{8}$. Sólo $49 \%$ del total de inmigrantes cuenta con cobertura de salud, observándose mayor desprotección de las mujeres en este ámbito, particularmente alto en el caso de población peruana ${ }^{9}$. 
De acuerdo a la literatura internacional, esta nueva realidad plantea retos específicos en relación a las necesidades y utilización de servicios de salud y un progresivo incremento de los casos de infección por VIH/SIDA ${ }^{10-13}$.

Las características clínicas y epidemiológicas de poblaciones inmigrantes y autóctonas infectadas con VIH han sido comparadas en países europeos, Estados Unidos de América (E.U.A.) e Israel ${ }^{14-21}$. Se han estudiado factores probables que determinan disparidades en cuanto a retraso en el diagnóstico, acceso y adherencia a tratamiento antiretroviral (TARV) y resultado clínico ${ }^{22-28}$. Se ha planteado la necesidad de estrategias de prevención distintas a la población autóctona en estos grupos ${ }^{29-34}$.

Este trabajo busca estudiar las características clínicas y sociodemográficas de los pacientes infectados con VIH inmigrantes latinoamericanos entre los años 2003 y 2013. Se compararon algunos resultados con población autóctona para establecer posibles diferencias.

\section{Métodos}

Estudio descriptivo, retrospectivo, realizado en la Unidad de Infectología del Hospital Clínico San Borja Arriarán, Fundación Arriarán (FA). FA atiende a pacientes adultos infectados por VIH, beneficiarios del Servicio de Salud Metropolitano Central. Fundación Arriarán ha atendido desde su creación a más de 4.000 pacientes, de los cuales se encontraban en control médico activo al momento de este estudio 2.750 .

Se estudiaron todos los pacientes de nacionalidad distinta a la chilena, de origen latinoamericano, atendidos en dicha unidad en los últimos 10 años. Se excluyeron los pacientes en los que fue imposible acceder a la ficha clínica. Cada paciente extranjero fue pareado con un control nacional. Para esto se seleccionó al paciente de nacionalidad chilena con número de ficha inmediatamente superior al número de ficha del paciente extranjero seleccionado. En FA los pacientes se registran con número de ficha correlativo en base a la fecha de ingreso. De esta forma se garantiza que cada extranjero y su par nacional se corresponden en fecha de ingreso y por lo tanto se presume similar acceso a TARV, controles y manejo clínico.

Se recogieron las siguientes variables:

- Variables socio demográficas: País de origen, fecha de ingreso a controles en FA, sexo, escolaridad, comuna de residencia, fecha de nacimiento, edad de ingreso, consumo de alcohol, tabaco y drogas, orientación sexual.

- Variables de diagnóstico de infección por VIH: Año de notificación del Instituto de Salud Pública (ISP), fecha de ingreso a FA, mecanismo de contagio.

- Variables clínicas: Etapa CDC (Centros para el Control y Prevención de Enfermedades, E.U.A.), cantidad y tipos de infecciones de transmisión sexual (ITS) previo al diagnóstico de infección por VIH, enfermedad(es) marcadora(s) de SIDA, situación actual (controles activos, en abandono, traslado a otro centro, fallecido).

- Variables analíticas (al ingreso a la unidad): Serología de toxoplasmosis, hepatitis $\mathrm{B}$ y $\mathrm{C}$, test de tuberculina (PPD), prueba serológica para sífilis (VDRL) y enfermedad de Chagas.

- Variables sobre seguimiento: fecha de confirmación en el ISP, tiempo entre la fecha de confirmación e ingreso a controles médicos en FA, recuento de linfocitos CD4+ (LCD4+) basal (al ingreso a FA), a los 3 meses (o al primer control) y luego de 4 meses de iniciada la TARV, carga viral (CV) a 3 meses (o al primer control) desde iniciada la TARV, fecha del primer control y adherencia a TARV.

Algunas variable fueron eliminadas del análisis final por el gran porcentaje de valores omitidos en los registros, a saber: consumo de alcohol, tabaco y drogas recreacionales (dato disponible en la ficha clínica $<80 \%$ ), serología de enfermedad de Chagas (que no es solicitada en forma rutinaria, registro $<70 \%$ ) y serología de hepatitis C. PPD y serología de toxoplasmosis no se analizaron mayormente por la diferencia importante en el número de registros entre ambos grupos, que no permitió establecer asociaciones. Se recogió la mayor parte de la información desde bases informatizadas en FA. Los datos no disponibles en estas bases electrónicas se obtuvieron de la revisión de las fichas clínicas de los pacientes.

\section{Definiciones}

- Consumo de alcohol, tabaco y drogas: se dicotomizó esta variable interpretando negativo el auto-reporte de consumo "nunca", "0", "no". Cualquier reporte distinto (ocasional, a veces, consumo excesivo) fue interpretado como positivo.

- Orientación sexual: se registraron los resultados: heterosexual, homosexual, bisexual y transgénero. Para el análisis se dicotomizaron los datos obtenidos en "heterosexuales" y "hombres que tienen sexo con hombres (HSH)" agrupando en esta última categoría a homo y bisexuales y transgénero. Las mujeres se agruparon en la categoría "heterosexual", ya que no hubo reportes de otra orientación.

- Control activo: paciente cumple con control médico en forma habitual en FA.

- Abandono: paciente sin control en FA > 1 año si no ha iniciado TARV y $>6$ meses en pacientes con TARV.

- Traslado: paciente solicitó y tramitó traslado a otro centro para continuar sus controles.

- Test de tuberculina (PPD): se consideraron positivos valores mayores a $5 \mathrm{~mm}$ de induración.

- Carga viral: para los análisis se expresó en valores de 


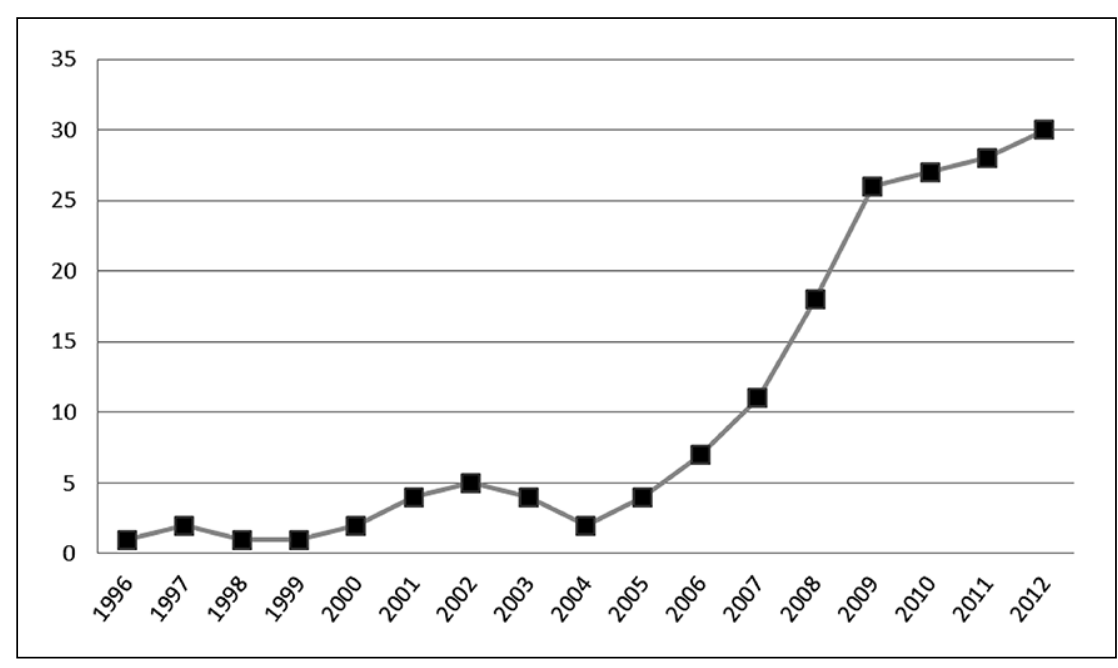

Figura 1. Distribución de inmigrantes por año de ingreso a Fundación Arriarán. Cantidad de sujetos ingresados anualmente en número absoluto.

\begin{tabular}{|lcc|}
\multicolumn{4}{c}{$\begin{array}{c}\text { Tabla 1. Distribución de Inmigrantes por país de } \\
\text { procedencia, detalle en número absoluto y porcentual. } \\
\text { Fundación Arriarán }\end{array}$} & 2003-2013 \\
\hline Nacionalidad & $\mathbf{n}$ & $\%$ \\
Perú & 96 & 55,2 \\
Colombia & 21 & 12,1 \\
Cuba & 11 & 6,3 \\
Ecuador & 11 & 6,3 \\
Argentina & 9 & 5,2 \\
Brasil & 7 & 4,0 \\
Haití & 4 & 2,3 \\
Venezuela & 4 & 2,3 \\
República Dominicana & 3 & 1,7 \\
Bolivia & 2 & 1,1 \\
México & 2 & 1,1 \\
Paraguay & 2 & 1,1 \\
Panamá & 1 & 0,6 \\
Uruguay & 1 & 0,6 \\
Total & 174 & 100 \\
\hline
\end{tabular}

$\log 10$. Los valores indetectables (LDE) se analizaron considerando como tal el valor de acuerdo a la técnica en uso del momento.

- Adherencia al año de TARV: Se evaluaron los registros de farmacia en los últimos 100 días, asignándose un punto porcentual a cada día de retraso o falta en el retiro de medicamentos. Alternativamente, se revisaron los registros en la ficha clínica de cada paciente y se consignó el porcentaje registrado por los médicos tratantes en el control más cercano al año de TARV. Se consideró como "sub óptimo" cualquier valor distinto de $100 \%$.

Se asumió una distribución normal de la población. Los datos continuos se presentan en medianas y desviación estándar y la comparación entre grupos se realizó con prueba de $\mathrm{T}$ de Student. Los datos categóricos se presentan como números absolutos y porcentajes y la prueba de $\chi^{2}$ se utilizó para la comparación entre grupos. Se definió como estadísticamente significativo un valor de $\mathrm{p}<0,05$. Los análisis se realizaron con el software GraphPad Prism 5.0 para Windows.

El protocolo fue presentado y aprobado por el Comité Ético Científico del Servicio de Salud Metropolitano Central.

\section{Resultados}

En la Unidad de Infectología del Hospital Clínico San Borja Arriarán, FA, se atendieron entre los años 2003 y 2013, 174 pacientes extranjeros de origen latinoamericano (4\% del total de pacientes activos a la fecha de realización de este estudio). Se incluyó para comparación un total de 174 pacientes nacionales. La distribución por años del número de ingresos de pacientes extranjeros demuestra un aumento evidente a lo largo del período de estudio con punto de inflexión en el año 2008 (Figura 1).

La distribución por país de procedencia se muestra en la Tabla 1. El país que aportó el mayor número de inmigrantes fue Perú (55\%), seguido de Colombia (12\%), Cuba y Ecuador ( $6 \%$ cada uno). Otras países de origen fueron Argentina (5\%), Brasil (4\%), Haití (2\%), Republica Dominicana (2\%) y Venezuela (2\%). Bolivia, México, Panamá, Paraguay y Uruguay tuvieron una representación de $1 \%$ cada uno.

Las características basales para ambos grupos se describen en la Tabla 2.

La distribución por sexo mostró diferencias estadísticamente significativas. Para pacientes extranjeros, $26 \%$ correspondió a sexo femenino, mientras que los chilenos presentaron $9 \%$ de sexo femenino $(\mathrm{p}<0,0001)$ (Figura 2). La mediana de edad al ingreso a FA fue de $33,8 \pm 0,67$ años para extranjeros y de $35,2 \pm 0,86$ para pacientes nacionales (NS).

En cuanto a escolaridad, no se observaron diferencias significativas entre ambos grupos. Este dato no se objetivó en alrededor de $30 \%$ en ambos grupos. Entre los pacientes en que sí se consignó este dato, el grado de escolaridad más frecuente correspondió a "enseñanza media" (45\% para extranjeros y $31 \%$ para nacionales). El resto se distribuyó entre educación técnica y superior. El 10\% de los extranjeros y $15 \%$ de nacionales reportaron sólo educación básica. 


\begin{tabular}{|c|c|c|c|c|c|}
\hline & \multicolumn{2}{|c|}{ Extranjeros } & \multicolumn{2}{|c|}{ Nacionales } & \multirow{3}{*}{$\begin{array}{c}\mathbf{p} \\
<0,0001\end{array}$} \\
\hline Masculino & 128 & $74,0 \%$ & 152 & $87,0 \%$ & \\
\hline Femenino & 46 & $26,0 \%$ & 16 & $9,0 \%$ & \\
\hline Edad de ingreso (mediana años) & 32,1 & (IIC: 27,1-39,8) & 32,6 & $(26,5-41,6)$ & 0,2614 \\
\hline Conducta sexual & & & & & $<0,0055$ \\
\hline $\mathrm{HSH}$ & 108 & $62,1 \%$ & 126 & $72,4 \%$ & \\
\hline Heterosexual & 65 & $37,4 \%$ & 39 & $22,4 \%$ & \\
\hline Sin dato & 1 & $0,6 \%$ & 9 & $5,2 \%$ & \\
\hline \multicolumn{6}{|l|}{ Mecanismo de contagio } \\
\hline Sexual & 172 & $98,8 \%$ & 170 & $97,7 \%$ & \\
\hline Otro & 1 & $0,6 \%$ & 2 & $1,2 \%$ & \\
\hline Sin dato & 1 & $0,6 \%$ & 2 & $1,2 \%$ & \\
\hline \multicolumn{6}{|l|}{ Clasificación CDC de ingreso } \\
\hline SIDA & 55 & $31,6 \%$ & 63 & $36,2 \%$ & \\
\hline No SIDA & 98 & $56,3 \%$ & 93 & $53,4 \%$ & \\
\hline Sin dato & 21 & $12,1 \%$ & 18 & $10,3 \%$ & \\
\hline CD4 basal (mediana) & 286 & (IIC: $170,5-432,5)$ & 287 & (IIC: 93-496) & 0,2318 \\
\hline Tiempo de latencia entra confirmación e ingreso en meses (mediana) & 3,1 & (IIC: $1,5-9)$ & 3,5 & (IIC: $1,8-13,4)$ & 0,1775 \\
\hline
\end{tabular}

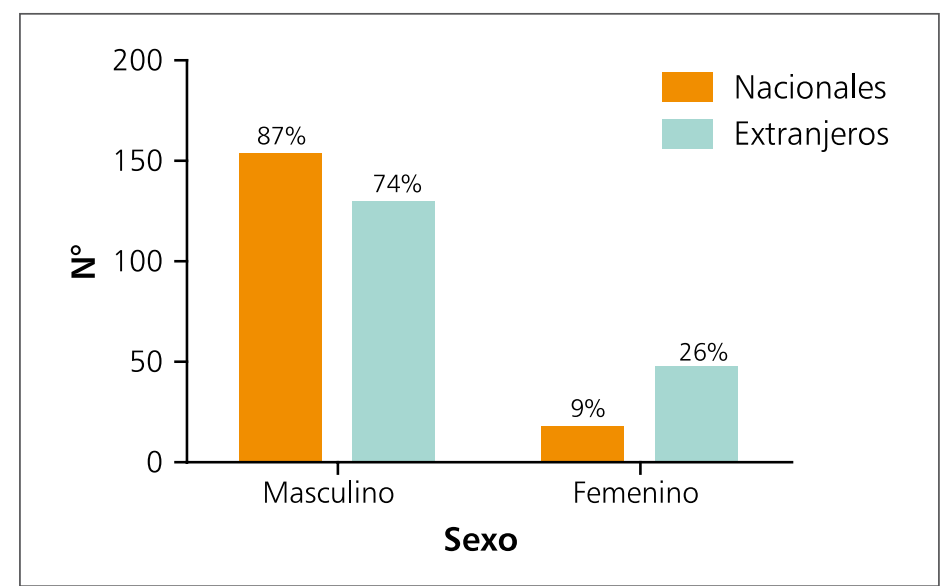

Figura 2. Distribución por sexo en ambos grupos, número absoluto. Fundación Arriarán 2003-2013.

La comuna que más registró ingresos a la Unidad de Infectología desde el sector Metropolitano Central de Santiago correspondió a Santiago Centro ( $77 \%$ de extranjeros y $58 \%$ de nacionales).

Con respecto a la orientación sexual, se observaron diferencias estadísticamente significativas entre ambos grupos. El porcentaje de pacientes con conducta heterosexual reportada correspondió a 37\% de los pacientes extranjeros y $22 \%$ de los nacionales, mientras que para HSH fue de $62 \%$ para extranjeros y $72 \%$ para nacionales, considerando a todo el universo de pacientes, sin diferenciar por sexo $(p<0,0055)$ (Figura 3). Al desagregar por sexo ambos grupos, presentan $15 \%$ de HSH para pacientes de sexo masculino.

El mecanismo de contagio fue sexual en la mayoría de los pacientes

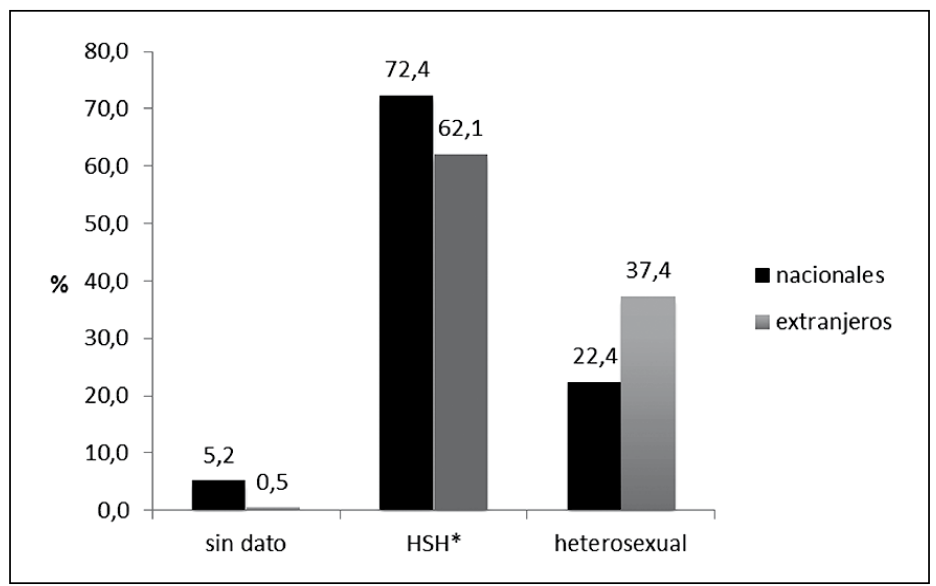

Figura 3. Conducta sexual en ambos grupos. Distribución porcentual. *Hombres que tienen sexo con hombres. Fundación Arriarán 2003-2013.

en ambos grupos ( $99 \%$ de los extranjeros y $98 \%$ de los nacionales). No hay registros sobre el país de contagio.

El $86 \%$ de los extranjeros y $77 \%$ de los nacionales se encontraban en control médico activo al momento del estudio.

Con respecto a las serologías de tamizaje al ingreso, se observó diferencia significativa entre los nacionales sin dato y los extranjeros sin dato $(\mathrm{p}<0,0001)$ para PPD, serología de toxoplasmosis y antígeno de superficie de hepatitis B (HBsAg). En todos los casos citados el número mayor de serologías se solicitó en el grupo de extranjeros.

Para HBsAg el porcentaje de positividad fue de $10 \%$ para extranjeros y $7 \%$ para nacionales.

El VDRL fue positivo en $11 \%$ de los pacientes extranjeros al ingreso 


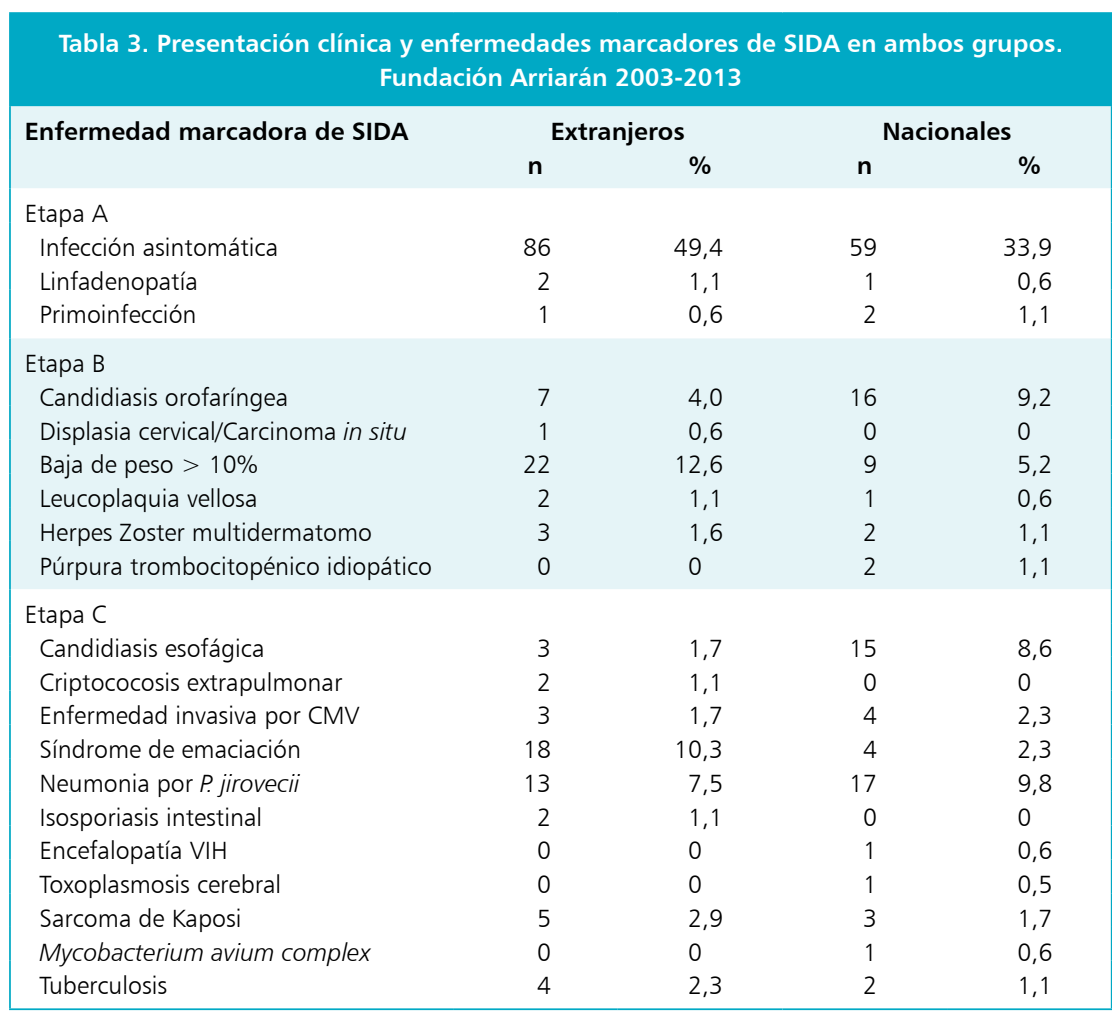

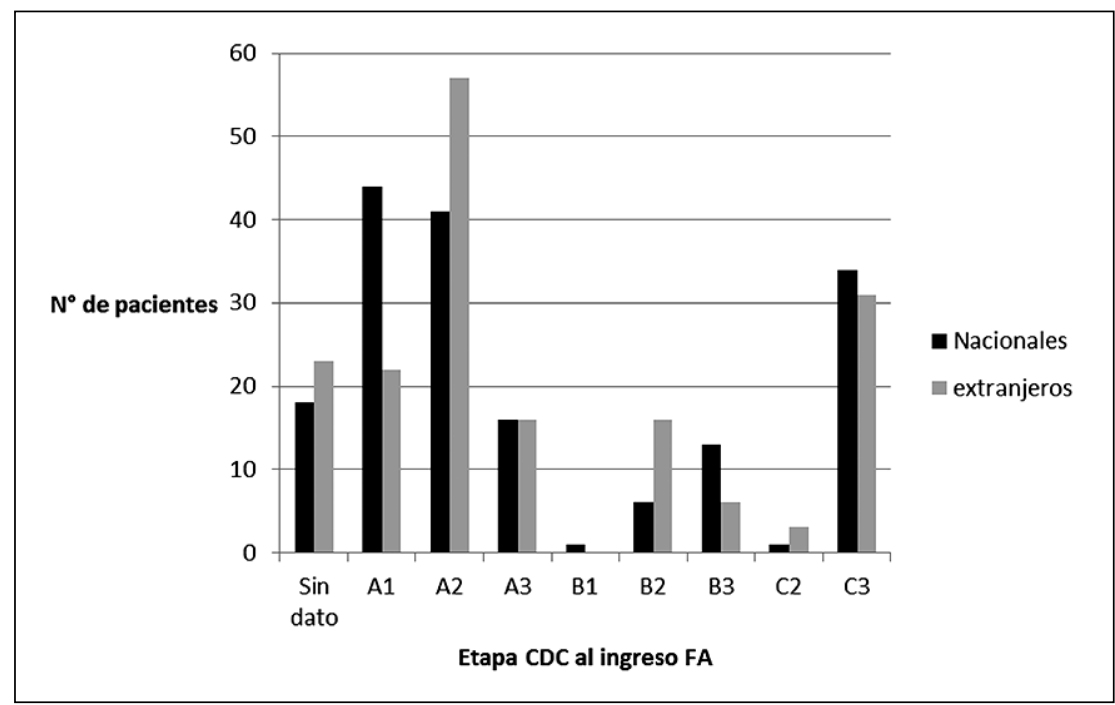

Figura 4. Etapa CDC al ingreso para ambos grupos. Fundación Arriarán 2003-2013.

y en $10 \%$ de los nacionales. Dato no disponible en $16 \%$ de los extranjeros y $42 \%$ de los nacionales.

Se estudio el número de ITS reportadas previo al ingreso a la FA. No se observaron diferencias estadísticamente significativas entre ambos grupos. El porcentaje de pacientes con al menos una ITS previa al diagnóstico de infección por VIH fue de $45 \%$ para nacionales y $41 \%$ en extranjeros.

Con respecto a las características clínicas, $49 \%$ de los extranjeros y $34 \%$ de los nacionales se presentaron al ingreso con infección en etapa CDC A (asintomáticos). La distribución de enfermedades en ambos grupos se presenta en la Tabla 3. No hubo diferencias significativas en relación a la etapa CDC de ingreso (Figura 4). El 31\% de los extranjeros (n: 55) y $36 \%$ de los nacionales (n: 63) se presentaron en etapa SIDA.

Si bien no corresponde a enfermedad marcadora de SIDA, en uno de los pacientes extranjeros se encontró un estudio parasitológico positivo para Strongyloides stercoralis, Blastocystis hominis y Entamoeba histolytica. En otro paciente se encontró Campylobacter spp. Otros dos se presentaron con fiebre tifoidea al ingreso.

Se recolectaron los antecedentes referidos a LCD4 basal y monitoreo de respuesta a TARV. La mediana de recuento de LCD4+ al ingreso a control fue de 286 céls/ $\mathrm{mm}^{3}$ (intervalo intercuartílico (IIC): 170-432, Rango intercuartílico (RQ): 262 para extranjeros y 287 céls $/ \mathrm{mm}^{3}$ para nacionales (IIC: 94-496; RQ: 402). El 83\% (n: 144) de los extranjeros y $66 \%$ (n: 116) de los nacionales se encontraban con TARV al momento del estudio. El primer control post inicio de TARV se realizó con una mediana de 5 meses (IIC: 4-7,2; RQ: 3,2) para extranjeros y 4,5 meses (IIC: $3,1-5,8 ;$ RQ: 2,7 ) para los pacientes nacionales. La mediana de recuento de LCD4+ al primer control fue de 316 céls $/ \mathrm{mm}^{3}$ (IIC: $214.5-414.5$; RQ: 200) para extranjeros y de 292 céls $/ \mathrm{mm}^{3}$ (IIC: 182-453; RQ: 270). A los 12 meses, fue de 349 céls $/ \mathrm{mm}^{3}$ (IIC: 216-519, RQ: 303 ) en extranjeros y 341 (IIC: 220,5-474,5; RQ:254) en chilenos. La mediana de CV expresada en log 10 para fue de 1,3 (correspondiente LDE) para ambos grupos en ambos controles.

La adherencia fue sub-óptima en $28 \%$ de los pacientes en TARV para ambos grupos.

La mediana de tiempo entre la fecha de confirmación del ISP y el ingreso a FA fue de 3,1 meses (IIC 1,5-9 meses; RQ: 7,5) para los pacientes extranjeros y 3,5 para nacionales (ICC: 1,8-13,4; RQ: 11,6).

\section{Discusión}

Según los datos obtenidos en este trabajo, hubo un aumento creciente del ingreso de inmigrantes a FA a través de los años de observación, que podría explicarse por el aumento total de inmigrantes en el país, dato apoyado por la información de extranjería. Cabe plantear hipótesis alternativas para este crecimiento, por ejemplo si existe un aumento del contagio en inmigrantes, si ha habido factores que determinen una mejor utilización de los servicios de salud y mayor acceso a éstos, o si existe un aumento en la 
sensibilización de este grupo con respecto a la importancia de tamizaje para VIH a lo largo del tiempo.

La población de inmigrantes estudiados, correspondientes a $4 \%$ de la población total de pacientes, sobrerepresenta el total de inmigrantes del país según el censo nacional de 2002 (1,2\%). El punto de inflexión observado en el año 2008 se correlaciona con los datos nacionales globales de notificación de infección por VIH y podría explicarse, tanto por la implementación del sistema de notificación en línea, como por la ejecución de la auditoría ministerial que comenzó a realizarse desde el último trimestre del año 2008 y que ha continuado como proceso permanente ${ }^{35}$.

La distribución de países de origen de los inmigrantes no se corresponde con el total nacional, en que predominan los argentinos ${ }^{4}$, pero sí con el total de pacientes del Área Metropolitana de Santiago, en que la mayoría de los inmigrantes son peruanos ${ }^{5}$. El estudio evidenció un alto porcentaje de colombianos, en concordancia con la tendencia reflejada por datos de encuesta CASEN y extranjería con respecto a la población de inmigrantes Según el último informe del Ministerio de Salud en el quinquenio 2008-2011 se observó la misma tendencia para extranjeros infectados con el $\mathrm{VIH}^{35}$.

La gran heterogeneidad en relación a las comunas de origen de los pacientes nacionales contrasta con los extranjeros que tienden a agruparse en la comuna de Santiago Centro. Si bien el número es pequeño y esto podría no necesariamente marcar una tendencia real, reportes de la literatura han demostrado una propensión a la agrupación de inmigrantes por comunas ${ }^{5}$.

Pese a las disparidades socio-económicas descritas, no se encontraron diferencias profundas en cuanto a la presentación clínica de la infección por VIH. La cifra de LCD4+ de ingreso estaría en concordancia con datos nacionales y analizándolo en conjunto con la etapa CDC de ingreso y el tiempo entre la confirmación diagnóstica y el ingreso a la FA se puede descartar la preconcepción de la presentación de extranjeros en etapas más tardías de la enfermedad. La mediana de recuento de LCD4+ al momento del diagnóstico no varió entre ambos grupos.

Si bien en los dos grupos la presentación clínica más habitual fue infección asintomática, las enfermedades definitorias de SIDA (EDS) fueron diferentes entre los grupos. Entre los extranjeros se diagnosticaron algunas enfermedades no pertenecientes al grupo de EDS, de muy baja prevalencia en nuestro país, especialmente en lo relativo a causas de diarrea crónica. Esto pone de manifiesto que el aumento en el número de inmigrantes extranjeros en el tiempo conllevará la necesidad de capacitación por parte de equipos de salud nacionales en patologías prevalentes en los países de origen.

Resulta llamativo el número similar de casos de TBC como enfermedad marcadora de SIDA en ambos grupos, ya que la prevalencia de ésta en los países mayormente representados por los extranjeros estudiados es mayor que en Chile ${ }^{36}$. Es de notar que en este estudio hubo una diferencia significativa entre el número de PPD solicitados a pacientes nacionales y extranjeros favoreciendo a estos últimos ( $\mathrm{p}<0,0001)$, por lo tanto, esto no es atribuible a un sesgo diagnóstico.

Cabe destacar el alto número de ITS previo al diagnóstico de VIH en ambos grupos, que podría reflejar conductas de riesgo pese a las estrategias actuales y pone de manifiesto la necesidad de intensificar la prevención en ambos grupos.

En este estudio, las diferencias más significativas se encontraron en relación a la distribución por género y orientación sexual. Esto estaría en concordancia con los reportes de la literatura científica, en que a medida que aumenta la prevalencia de infección por VIH, tiende a haber mayor feminización de la población infectada y deja de hacerse marcada la diferencia entre población heterosexual y $\mathrm{HSH}^{37,38}$. Los países de origen de los inmigrantes corresponden a países de prevalencia media de infección por VIH (entre 2,5 y 4,4/1.000 habitantes), mientras que Chile corresponde a un país de baja prevalencia (entre $0,5$ y 2,4/1.000 habs. $)^{38}$. Estos datos plantean la necesidad de estrategias de prevención distintas a las adoptadas hasta ahora. Según los lineamientos ministeriales, se han definido estrategias basadas en poblaciones vulnerables prioritarias, entendidas como: $\mathrm{HSH}$, población homo/ bisexual, trabajadores(as) en comercio sexual, población transgénero y población privada de libertad ${ }^{39}$. El mayor número de mujeres debiese acarrear estrategias de prevención que las consideren en forma dirigida, tomando en cuenta dimensiones de género y sociales especificas para cada subgrupo. La mayor prevalencia en mujeres debiese también suponer un mayor énfasis en la prevención de la transmisión vertical.

Hay una diferencia estadísticamente significativa para la cantidad de serologías de toxoplasmosis y PPD solicitadas al ingreso que favorece a los extranjeros. Esto podría explicarse por un probable sesgo en los médicos que realizan la atención, pero la importancia de este tamizaje en todo paciente está ampliamente validada y es relevante destacar la necesidad de implementar políticas que hagan más eficiente la solicitud de éstas. También es preciso hacer énfasis en los grupos de tratantes en el registro adecuado del dato de consumo de alcohol, drogas, tabaco y escolaridad, antecedentes reconocidos en la literatura médica como relevantes en relación a la adherencia a $\mathrm{TARV}^{40}$, que pueden llevar a adoptar estrategias de manejo a priori de refuerzo de adherencia en ambos grupos.

El 28\% de los pacientes presentaron adherencia sub-óptima a TARV, lo que estaría en concordancia con reportes internacionales ${ }^{41}$. La respuesta virológica 
e inmunológica se corresponden con la experiencia clínica habitual y no hubo diferencias estadísticamente significativas entre ambos grupos. Esto podría significar que el acceso a la utilización del sistema de salud y las medidas de refuerzo de adherencia son similares. Sería interesante plantear un estudio futuro con un mayor número de pacientes y con análisis de subgrupos para evaluar esta condición, específicamente en pacientes de habla no hispana.

Este trabajo contribuye a visibilizar la situación de la población inmigrante en Chile. La literatura nacional en este ámbito es escasa y se puede suponer un incremento sostenido a futuro del número de inmigrantes. Este estudio permite además plantear variadas posibilidades de estudio futuros para contribuir en la mayor comprensión de la situación de este grupo vulnerable. Su diseño comparativo no permite establecer relaciones causales entre las variables estudiadas y el hecho de ser inmigrante. La aplicación del estudio en un solo centro de Santiago podría generar resultados sesgados por las diferencias de distribución de los inmigrantes en el territorio nacional, y más aún, en su distribución por comunas que limitan la extrapolación de los resultados a poblaciones mayores, limitando la validez externa del estudio. La segregación por comunas descrita en la literatura demuestra una alta concentración de población peruana en los sectores de residencia de la población atendida en nuestro centro, lo que determinaría, en contraste, una alta validez interna de éste. Al momento del estudio no se encontraban aún disponibles los informes del reciente censo de 2012, por lo que la realidad epidemiológica nacional descrita podría experimentar modificaciones en un futuro próximo.

Sería interesante realizar futuros estudios que incluyan datos tales como el status para infección por VIH previo al ingreso al país, las razones para realizar la serología, la percepción de los pacientes en cuanto a la calidad, oportunidad y percepción de discriminación en la atención de salud, y los factores socioculturales y de condiciones de vida en nuestro territorio que determinen manejos diferenciados, además de comparar datos referentes a respuesta a TARV, adherencia y tasas de abandono. Convendría analizar en estudios futuros las características socioeconómicas y contrastarlas con un análisis más profundo de subgrupos para identificar eventuales diferencias por países. Resulta relevante estudiar algunas de las hipótesis que se plantean en el trabajo, por ejemplo, si existe efectivamente un aumento del contagio en inmigrantes. Otras de las hipótesis a ser evaluadas en estudios de corte cualitativo son, por ejemplo, si ha habido factores que determinen cambios en el acceso y utilización de servicios de salud o si existe una mayor sensibilización en este grupo a la importancia del tamizaje de infección por VIH a lo largo del tiempo. La realización de estudios longitudinales permitiría evaluar la evolución de la realidad en el tiempo y entregar más luces al respecto.

\section{Resumen}

Introducción: Chile ha experimentado un aumento de inmigrantes, especialmente sudamericanos. La literatura médica internacional describe necesidades de atención diferenciadas entre migrantes y poblaciones autóctonas. En Chile se han mantenido estrategias de prevención de la infección por virus de inmunodeficiencia humana (VIH) únicamente basadas en necesidades de la población nacional. Objetivos: Describir las características clínicas y epidemiológicas de la infección por VIH en pacientes inmigrantes latinoamericanos atendidos en Fundación Arriarán en los últimos 10 años, comparados con población chilena. Métodos: Estudio retrospectivo. Comparación de características basales de población latinoamericana de nacionalidad distinta a la chilena y pacientes chilenos en la misma unidad. Resultados: Se observó un aumento gradual de casos en inmigrantes a lo largo del periodo. Las nacionalidades predominantes fueron peruana $(55 \%)$ y colombiana $(12 \%)$. Los extranjeros presentaron mayor proporción de mujeres (26 vs 9\%) y de conducta heterosexual ( 37 vs $22 \%$ ) que los nacidos en Chile. No hubo diferencias en la presentación clínica. Conclusiones: Hubo diferencias significativas en la distribución por género y conducta sexual de los pacientes extranjeros en comparación con los nacionales. Esto plantea la necesidad de adoptar estrategias de prevención con mayor énfasis en mujeres y población heterosexual en este subgrupo de pacientes vulnerables.

\section{Referencias bibliográficas}

1.- Gascon Brustenga J, Navarro Beltra M, Muñoz Sanz A, Mahillo Durán B. Epidemiologia del VIH/SIDA en el mundo y el fenómeno migratorio actual. Enferm Infecc Microbiol Clin 2008; 26 Suppl 5: 2-5.

2.- Desarrollo del fenómeno de las migraciones en Chile. Evolución de la gestión gubernamental desde 1990-2007. In: Migración DdEy, ed.
3.- Censos de población históricos. 2013. (Accedido el 31 de enero de 2013) http://www.ine.cl/ canales/usuarios/censos_digitalizados.php).

4.- Martínez Pizarro J. PyDdC, División de Población de la CEPAL. El encanto de los datos. Sociodemografía de la inmigración en Chile según el censo de 2002. In: desarrollo C-SPy, ed.: Naciones Unidas; 2003.

5.- Schiappacasse Cambiaso P. Segregación residencial y nichos étnicos de los inmigrantes internacionales en el Área Metropolitana de Santiago. Revista de Geografía Norte Grande 2008: 21-38.

6.- Solimano V T A. Migraciones internacionales en un contexto de crecimiento económico. El caso de Chile. In: CEPAL, ed. Santiago de Chile: Naciones Unidas; 2006

7.- Cabieses B, Tunstall H, Pickett K E, Gideon J. Understanding differences in access and use of healthcare between international immigrants to 
Chile and the Chilean-born: a repeated crosssectional population-based study in Chile. Int $\mathrm{J}$ Equity Health 2012; 11: 68.

8.- Politica migratoria en Chile. 2008. (Accedido el 22 de enero de 2013) http:// www.extranjeria.gov.cl/filesapp/seminario migraciones_13_11_08.pdf.)

9.- Cabieses B, Pickett K E, Tunstall H. What are the living conditions and health status of those who don't report their migration status? A population-based study in Chile. BMC Public Health 2012; 12: 1013.

10.- Jansà J M, García de Olalla P. Salud e inmigración: nuevas realidades y nuevos retos. Gaceta Sanitaria 2004; 18: 207-13.

11.- Hauck F R, Corr K E, Lewis S H, Oliver M N. Health and health care of African refugees: an underrecognized minority. J Natl Med Assoc 2012; 104: 61-71.

12.- Navarro Beltra M, López-Velez R. VIH e inmigracion: interseccion de dos conjuntos. Enferm Infecc Microbiol Clin 2008; 26 Suppl 5: 1.

13.- López-Velez R, Navarro Beltra M, Hernando Jerez A, del Amo Valero J. Infeccion por el VIH en inmigrantes. Enferm Infecc Microbiol Clin 2008; 26 Suppl 5: 12-21.

14.- Ramos J M, Gutiérrez F, Padilla S, Masia M, Escolano C. Caracteristicas clinicas y epidemiologicas de la infección por el VIH en extranjeros en Elche, Espana (1998-2003). Enferm Infecc Microbiol Clin 2005; 23 : 469-73.

15.- Sánchez M A, Lemp G F, Magis-Rodríguez C, Bravo-García E, Carter S, Ruiz J D. The epidemiology of HIV among Mexican migrants and recent immigrants in California and Mexico. J Acquir Immune Defic Syndr 2004; 37 Suppl 4: S204-14.

16.- Llenas-García J, Rubio R, Hernando A, Fiorante S, Maseda D, Matarranz M, et al. Características clínico-epidemiológicas de los pacientes inmigrantes con infeccion por el VIH estudio de 371 casos. Enferm Infecc Microbiol Clin 2012; 30: 441-51.

17.- Bouckenooghe A R, Shandera W X. The epidemiology of HIV and AIDS among Central American, South American, and Caribbean immigrants to Houston, Texas. J Immigr Health 2002; 4: 81-6.

18.- Jerez A H, García-Cerrada C, Ortega F P, García R R, Gómez R H, González C C, et al. Infeccion por el VIH en inmigrantes: diferencias clínico-epidemiológicas con la población autóctona en un area de salud de la Comunidad de Madrid (2001-2004). Enferm Infecc Microbiol Clin 2007; 25: 441-5.
19.- Thierfelder C, Weber R, Elzi L, Furrer H, Cavassini M, Calmy A, et al. Participation, characteristics and retention rates of HIVpositive immigrants in the Swiss HIV Cohort Study. HIV Med 2012; 13: 118-26.

20.- Pollack S. Epidemiological and immunological study of HIV-seropositive Ethiopian immigrants in Israel. The Israel AIDS Study Group. Isr J Med Sci 1993; 29: 19-23.

21.- Manfredi R, Calza L, Chiodo F. HIV disease among immigrants coming to Italy from outside of the European Union: a case-control study of epidemiological and clinical features. Epidemiol Infect 2001; 127: 527-33.

22.- Dang B N, Giordano T P, Kim J H. Sociocultural and structural barriers to care among undocumented Latino immigrants with HIV infection. J Immigr Minor Health 2012; 14: 124-31.

23.- Keesee M S, Natale A P, Curiel H F. HIV positive Hispanic/Latinos who delay HIV care: analysis of multilevel care engagement barriers. Soc Work Health Care 2012; 51: 457-78.

24.- Kang E, Rapkin B D, Springer C, Kim J H. The "Demon Plague" and access to care among Asian undocumented immigrants living with HIV disease in New York City. J Immigr Health 2003; 5: 49-58

25.- Nkulu Kalengayi F K, Hurtig A K, Ahlm C, Krantz I. Fear of deportation may limit legal immigrants' access to HIV/AIDS-related care: a survey of Swedish language school students in Northern Sweden. J Immigr Minor Health 2012; 14: 39-47.

26.- Simbiri K O, Hausman A, Wadenya R O, Lidicker J. Access impediments to health care and social services between Anglophone and Francophone African immigrants living in Philadelphia with respect to HIV/AIDS. J Immigr Minor Health 2010; 12: 569-79.

27.- Rosenthal L, Scott D P, Kelleta Z, Zikarge A, Momoh M, Lahai-Momoh J, et al. Assessing the HIV/AIDS health services needs of African immigrants to Houston. AIDS Educ Prev 2003 15: $570-80$.

28.- Othieno J. Understanding how contextual realities affect African born immigrants and refugees living with HIV in accessing care in the Twin Cities. J Health Care Poor Underserved 2007; 18: 170-88.

29.- McMahon T, Ward P R. HIV among immigrants living in high-income countries: a realist review of evidence to guide targeted approaches to behavioural HIV prevention. Syst Rev 2012; 1: 56.

30.- Bouchaud O, Cherabi K. Caring for immigrants living with HIV-AIDS in France. Rev Prat 2006;
56: $1005-10$

31.- Cobos D G, Jones J W. Promoting passage through care: how to best serve undocumented immigrants with HIV. J Assoc Nurses AIDS Care 2008; 19: 320-4.

32.- Kuznetsov L, Matterne U, Crispin A, Ruzicka T, Zippel S A, Kuznetsov A V. Knowledge, attitude and behavioral intention to act regarding HIV infection and prevention in immigrants from the former Soviet Union in Germany: A comparative study with the native population. J Immigr Minor Health 2013; 15(1): 68-77.

33.- Ruiz M, Briones-Chávez CS. How to improve the health of undocumented Latino immigrants with HIV in New Orleans: an agenda for action Rev Panam Salud Publica 2010; 28: 66-70.

34.- Mechali D, Bouchaud O. Should there be a specific management for HIV infectedimmigrants in France?. Med Mal Infect 2008; 38: 438-42.

35.- Salud Md. Informe Nacional "Evolución VIH SIDA, Chile 1984 - 2011". In: Departamento de Epidemiología Ddps, ed. Santiago: MINSAL; 2012.

36.- WHO. Global Tuberculosis Report. In: Organization WH, ed.: World Health Organization; 2012.

37.- García C R N P J, Bayer A M, Cárcamo C P, Lazo M, La Rosa S, Mallma P S, et al. VIH y vulnerabilidad de la mujer en el Perú: evidencias y recomendaciones para la acción. In. Lima: Universidad Peruana Cayetano Heredia, Facultad de Salud Pública y Administración, Unidad de Epidemiología, ETS y VIH; UNFPA/ Fondo de Población de las Naciones Unidas, ; 2013

38.- Teva I, Bermúdez M P, Ramiro M T, Buela-Casal G. Current epidemiological situation of HIV/AIDS in Latin America: analysis of differences among countries. Rev Med Chile 2012; 140: 50-8.

39.- Políticas y estrategias de prevención. Ministerio de Salud de Chile. (Accedido 22 enero, 2013, en http://www.redsalud.gov.cl/archivos/vih/ Documento Trabajo_vulnerabilidad.pdf.)

40.- O'Connor J L, Gardner E M, Mannheimer S B, et al. Factors associated with adherence amongst 5295 people receiving antiretroviral therapy as part of an international trial. J Infect Dis 2013; 208: 40-9.

41.- Kasparas PRPG, Ravasi G, Sedlacek D, Coqui T. Experiencias exitosas en el manejo de la adherencia al tratamiento antirretroviral en Latinoamérica. In: Salud. OPdl, ed. Washington, D. C: Organización Panamericana de la Salud.; 2011. 
Pueden emplearse desde su primera mención en el texto, tablas y figuras. Sin embargo, use el término completo en los títulos y en su primera cita en resúmenes.

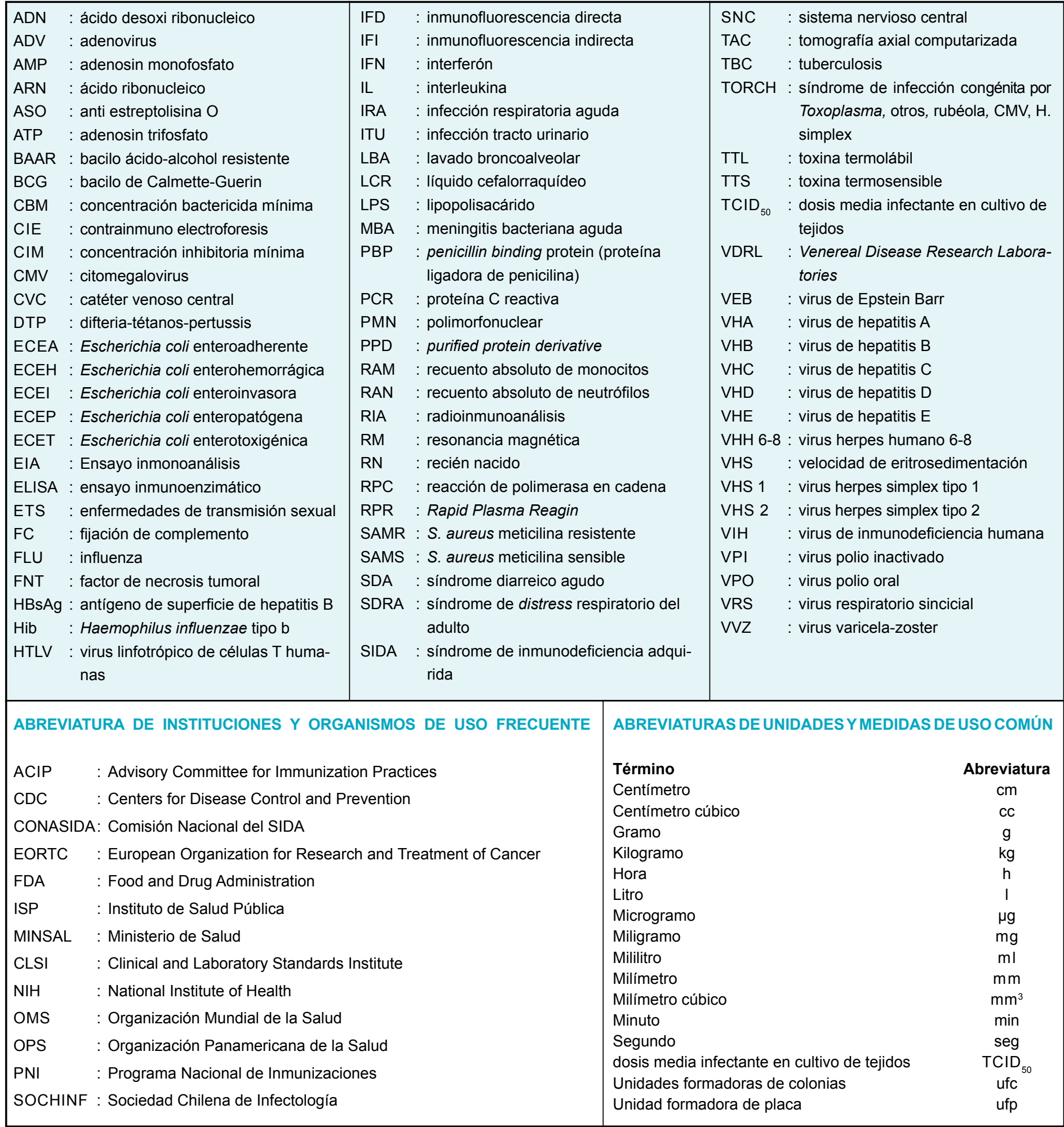

El Comité Editorial acogerá favorablemente la sugerencia de otras abreviaturas enviadas por los lectores. 